\title{
El adolescente procesado en el sistema de justicia de México: ¿delincuente amateur?
}

\section{Arturo Alvarado Mendoza*}

\section{Resumen}

Las transgresiones a las leyes y códigos penales cometidas por adolescentes en nuestra región son un problema público que merece mayor atención. Constituyen una base documental única para conocer las tendencias, grados y características de los delitos, y los perfiles de las y los transgresores menores de edad. Este trabajo procura construir el complejo perfil del joven infractor a partir de datos de menores de edad procesados por el sistema de justicia de adolescentes en México. Propone que la conducta obedece a una doble interacción entre las tensiones producidas por la limitada estructura de oportunidades que viven los jóvenes así como por una construcción pública y mediática de un perfil de sujetos propensos a cometer delitos, sustentada en la costumbre de estrategias de control policial y judicial, con prácticas que etiquetan e institucionalizan un solo perfil de adolescentes. Revisa críticamente las teorías sociológicas de la desviación y del comportamiento delictivo juvenil. Muestra los límites de esta categoría y propone algunas alternativas.

Palabras clave: delincuente amateur, institucionalización de adolescentes, delincuencia juvenil.

* El Colegio de México, Ciudad de México, México. 


\section{The adolescent prosecuted in the Mexican justice system: amateur criminal?}

\section{Abstract}

Adolescents' transgressions to laws in the Latin American region are a public problem that needs more attention. This article analyzes its complex characteristics, its sociodemographic and delinquent profile in Mexico, based on data of juvenile population treated by the Adolescent Justice System. The Article develop two propositions explaining juvenile delinquency. It is the result of an interaction between the social tensions produced by a limited opportunity structure, as well as an outcome of the systematic, unequal profiling and labelling of police and judicial authorities of this disadvantaged group. The article proposes a critical reassessment of the category of amateur delinquent within the sociological and criminological literature.

Keywords: Juvenile delinquency, amateurs, institutionalization of adolescents.

\section{Presentación}

E I propósito del trabajo consiste en discernir cuál es la mejor forma de comprender la conducta transgresora de la ley en los jóvenes menores de edad. Si bien distintos agentes policiales y del poder judicial, así como los medios de comunicación y asociaciones civiles mencionan que la participación de los jóvenes en actos delictivos y en organizaciones criminales está aumentando, no hay datos públicos suficientes y consistentes que puedan corroborar estas ideas. Tampoco son claros el perfil ni el motivo por el cual cometen estas infracciones. Ante la falta de información, cabe formular algunas interrogantes. ¿El comportamiento de estos jóvenes presenta las características de la delincuencia amateur? ¿Esta conducta es parte constitutiva de una trayectoria de vida asociada con una carrera criminal que construyen estos sujetos? o, por el contrario, ¿son víctimas y producto de procesos de selección policial o judicial (profiling), producidos por instituciones de control? 
La literatura tiene varias conjeturas con las cuales intenta explicar el delito juvenil, pero no hay un paradigma común. Ahora bien, hay ciertos acuerdos sobre las características del comportamiento considerado desviado (de la ley). Entre todos ellos, la edad constituye un componente central en la caracterización de este tipo de comportamiento adolescente, esto es el de personas entre los 12 y los 18 años. Según Tenenbaum (2020) y Kessler (2010), los estudios de "la curva de la edad del delito" demuestran que el comportamiento delictivo de la mayoría de los jóvenes es ocasional o responde a un tránsito en el curso de sus vidas. Pero, como se muestra en el caso de México, este comportamiento no siempre es "ocasional", por lo menos para una porción de la población procesada por el sistema de justicia penal. Por el contrario, muchos individuos, incluso los clasificados y procesados como primo-delincuentes, muestran conductas reiteradas de infracción a las leyes. Buena parte de la literatura procura comprender y encuadrar el comportamiento siguiendo interpretaciones como la teoría de las actividades rutinarias y de la oportunidad de cometer el delito (Cohen; Felson, 1979); otros utilizan el enfoque de una subcultura del delito o el labelling approach o de las características psicosociales del joven delincuente (Merton, 1938; Becker, 1963; Matza; Sykes, 1961; Kessler, 2010; Tenenbaum, 2020).

La evidencia en América Latina para comprobar algunas de estas conjeturas es dispersa y ofrece una visión fragmentada. Dentro de la literatura y en los registros públicos hay una carga enorme de datos sobre los jóvenes infractores de barrios "populares" o de clases sociales "bajas", pero poco sabemos de otros grupos sociales en barrios con mejores condiciones. Tampoco se ha hecho suficiente investigación sobre el papel que juegan las instituciones públicas de control y castigo en la focalización de su trabajo y el etiquetamiento de este subgrupo de jóvenes. El castigo contínua siendo la base y solución a los conflictos y problemas producidos por los adolescentes.

Este artículo propone hacer énfasis en la manera que operan las instituciones de control que focalizan y criminalizan a los adolescentes con 
ciertos perfiles sociodemográficos/socioespaciales, así como al contexto en donde ocurre el delito. Además, propone revisar algunas tesis de los estudios sobre la delincuencia amateur a partir de un examen crítico de las dimensiones con las que explican el comportamiento delictivo juvenil. No sólo es cuestión de una agencia con una construcción psicosocial específica; tampoco es solamente vivir bajo una subcultura del delito. En este trabajo proponemos que obedece a una doble interacción entre las tensiones producidas por la limitada estructura de oportunidades que viven los jóvenes, así como por una construcción pública y mediática de un perfil de sujetos propensos a cometer delitos, construida por una larga costumbre de estrategias de control policial y judicial, con prácticas que capturan, etiquetan e institucionalizan este tipo adolescentes. Estas dos propuestas serán las hipótesis que guiarán el análisis y la explicación del caso mexicano.

Por lo tanto, se toman en cuenta las proposiciones de la literatura sobre el delito juvenil y, en particular, sobre el delito de adolescentes o menores de edad, que es clasificado de distintas formas en América Latina y para los cuales se han creado instituciones de justicia penal especializadas. Pero también hay un cuestionamiento hacia las propuestas de la literatura, para revisar si comprenden cabalmente un grupo de población adolescente que delinque bajo condiciones culturales y sociales "específicas" de México. ¿Son todos ellos sujetos propensos en lo individual y en su perfil social a cometer infracciones, o son también personas "orilladas" al delito (Tenenbaum, 2020), a la transgresión - etiquetadas, criminalizadas y perseguidas de manera desproporcional por las instituciones policiales o por el "trato preferencial de la policía y las cortes" (Matza; Skyes, 1961, p. 718)? Adicionalmente, nos preguntamos si quienes han sido aprehendidos, capturados por la policía y el sistema de justicia y, en particular, por el sistema penal de adolescentes ison una muestra representativa de la población juvenil o sólo un grupo "problemático"? Este trabajo propone que es la combinación de ambas y postula que hay un perfil de adolescentes capturados y convertidos en sujetos de tratamiento policial y penal por 
parte del Estado, esto es, pacientes de las instituciones coercitivas y de castigo. Esto encubre, vela la posibilidad de conocer de manera más completa el universo social y de clase dentro del cual emergen ciertos sujetos con conductas transgresoras.

Para analizar y construir una clasificación - con miras a una tipología - se consideran cinco dimensiones, a saber: i) la edad; ii) los perfiles sociodemográficos; iii) los delitos producidos (tipos, frecuencia y forma en que se cometen los actos ilícitos en forma individual o colectiva, como parte de crimen organizado; como rutinas, como oportunidades o actos planeados); iv) la relación con la autoridad familiar-parental-adulta (porque en el sistema penal juvenil mexicano la condición familiar es muy importante); v) las narrativas alrededor de los actos delictivos de algunos adolescentes.

El perfil sociodemográfico de los infractores en México está concentrado en un solo grupo que suma varias características propias de la exclusión y marginación social. La relación con la autoridad familiar-parental-adulta es muy importante, tal vez más que las relaciones entre pares en los barrios donde habitan. Las narrativas sobre los actos delictivos de algunos adolescentes muestran varios aspectos importantes en la construcción del sujeto infractor, entre ellos, el discernimiento sobre los actos criminales, la construcción y justificación de la víctima como merecedora de la agresión; el apego o no a valores sociales dominantes y las expectativas personales. Los valores sociales que expresan son heterogéneos. Algunos jóvenes procesados tienen aspiraciones asociadas con imaginarios de criminales "ejemplares" en México, pero otros también esperan ser sujetos trabajadores aceptados por la comunidad, queridos y respetados.

La conclusión a la que llega este trabajo es que el sistema de justicia penal mexicano capta un grupo selecto de jóvenes que no sólo cubre la categoría de delincuente amateur, sino que presenta rasgos de trayectorias de una carrera delictiva (combinada con otras actividades) incompletos para entender de manera íntegra el problema. Los rasgos sociodemográficos y psicológicos de esta población "cautiva" son más diversos que los que ha señalado la literatura, en particular por sus trayectorias personales desde 
su infancia, por la relación con los padres y por el contexto barrial en donde habitan.

\section{Método}

El trabajo analiza los principales tipos promedio de jóvenes que son procesados por el Sistema Integral de Justicia Penal de Adolescentes (SJIPA) en México. La información proviene de los registros públicos de justicia penal y de las estadísticas delictivas de México, así como de exploraciones de varios autores y entrevistas propias a adolescentes en los centros de tratamiento especializado o "comunidades", considerando componentes por grupos de edad, género, región, cantidad y tipo de delito. Igualmente, se recurre a fuentes documentales y de prensa; visitas a centros de tratamiento en Ciudad de México y otros países (Brasil, Colombia y Guatemala). ${ }^{1}$

\section{El problema y la discusión de la literatura para la región de A. L.}

Las transgresiones a las leyes y códigos penales cometidas por adolescentes en nuestra región son un problema público que requiere mayor atención por parte de los expertos. Constituyen una base documental única para conocer no sólo las tendencias, grados y características de los delitos, sino también los rasgos de los transgresores adolescentes, en particular los menores de edad. Buena parte de la literatura criminológica y jurídica los define y etiqueta como primo delincuentes; otra más como delincuentes amateurs, aficionados, y establece un perfil sociodemográfico, haciendo énfasis en la edad y algunos factores de riesgo, pero sin considerar la forma en que estos son etiquetados por el sistema; esto es, como son construidos como sujetos desviantes o peligrosos, proclives a cometer delitos, por las

1 El trabajo es parte de investigaciones previas sobre justicia juvenil, cuyo producto más reciente es el libro Los desafíos de la justicia de adolescentes en América Latina (en prensa). El reto es transformar los viejos sistemas punitivos con tratamientos sociopedagógicos y eliminar la violencia estatal de la que son víctimas los jóvenes o, como lo proponemos, cambiar las leyes en conflicto con los jóvenes. La edad es central para clasificar a esta población e imponerle responsabilidad penal. En la mayoría de los países, la edad mínima de responsabilidad es de 12 años, pero en Suriname, es de 10 años. 
instituciones de castigo público (las policías, las cortes y los medios de comunicación) por el poder adultocéntrico, por el pánico moral que los criminaliza y etiqueta (Cohen et al., 1972; Garland, 2008). Pero no hay un consenso respecto a lo que debe considerarse como delincuente juvenil o amateur. Para algunos la delincuencia juvenil es producto de rasgos psicológicos individuales, de disturbios-distorsiones de la personalidad o de conflictos emocionales del individuo; para otros es producto de la exposición de personalidades normales a un ambiente distorsionado, particularmente en la forma de una cultura desviante en la que algunos individuos menores de edad aprehenden a delinquir y otros a obedecer la ley (Matza; Sykes, 1961). Varios paradigmas guían la investigación (Misse; Motta, 1979). Para la criminología crítica de izquierda, el delito es "una declaración de rebeldía y protesta contra el sistema capitalista y liberal" (Pegoraro, citado en Tenenbaum, 2020, p. 24). Pero pocos estudios examinan el problema desde una perspectiva de derechos y privaciones. Hay algunos rasgos que comparte la literatura de estos individuos como sujetos desviados. Entre los autores más influyentes sobre estos temas en la región están la teoría de la tensión social de Merton (1938) y la privación relativa. También están Howard Becker (1963), con su interpretación de las oportunidades de cometer delitos, así como por el etiquetamiento; David Matza y Gresham Sykes, con la teoría de los valores subterráneos y de la neutralización. Según Tenenbaum (2020), es notorio el uso de la teoría de las actividades rutinarias, del control social o de la oportunidad de Lawrence Cohen y Marcus Felson (1979). Hay un proceso de personas que cometen transgresiones a la ley; hay una construcción pública del sujeto llamado menor infractor, primodelincuente o delincuente amateur, que está estructurada a partir de normas, opiniones, valores dominantes por grupos de poder (Becker, 1963; Matza; Sykes, 1961). Considerar estos sujetos como delincuentes, transgresores o infractores, detona lo que algunos autores enumeran como el conjunto de mecanismos de acción que motivan las detenciones policiales y posteriormente su procesamiento por las instituciones de justicia penal (Matza; Skyes, 1961). Pero, en realidad, el etiquetamiento se concentra en un perfil sociodemográfico. De acuerdo 
con Tenenbaum (2020, p, 23), estamos frente a niñas, niños y adolescentes con "vidas castigadas, [...] signadas por [...] construcciones arbitrarias, con desventajas y exclusiones [...] múltiples".

Este trabajo considera tres tipos de jóvenes; en primer lugar, el delincuente menor de edad como parte de este tipo de delincuente juvenil, ya sea primo delincuente, amateur o un transgresor con trayectoria. El grupo de estudio es aquel que ha sido aprehendido por el sistema de policial y procesado por el de justicia. La edad en la cual comienzan a delinquir es relativamente temprana y presenta varias características de una conducta desviada. En segundo lugar, está el delincuente amateur. Algunos de los jóvenes procesados ensayaron por primera vez lo que es la transgresión a las normas, como también aquellos que ya tienen una idea más definida de la comisión de delitos y han repetido esta conducta de manera individual o colectiva. En tercer lugar, está el agresor con trayectoria, con experiencia, estrategias, narrativas y valores más asociados con una carrera delictiva.

\section{Contexto regional}

En la gran mayoría de los países de América Latina opera un sistema penal especializado para los adolescentes que han sido reformados, pero siguen una trayectoria dependiente de regímenes punitivos y autoritarios. El castigo continua siendo la solución a los conflictos y problemas producidos por los adolescentes. La población captada por los sistemas de justicia para adolescentes parte de un conjunto de parámetros comunes. Los rangos de edad abarcan personas entre los 10 y hasta más de 18 años, aunque la mayoría se encuentra entre los de las edades entre 16 y 17. Además, los sistemas tienen procesado a un grupo de mayores de edad que cometieron delitos como adolescentes, pero fueron aprehendidos y juzgados posteriormente y todavía estaban cumpliendo las medidas dictadas por los jueces (en México la pena máxima es de 5 años).

La información disponible es escasa, mas algunos trabajos como el de Vernazza (2016) ofrecen estimaciones generales. Los datos que se presentan aquí no constituyen una muestra representativa de la población recluida; es 
ilustrativa de la variedad de casos, delitos y edades (cuadro 1). Algunos temas destacan. En primer lugar, las tasas de adolescentes privados de libertad en cinco países son más altas que el promedio, pero Uruguay, Perú, Brasil y Chile tienen tasas desproporcionalmente más altas al resto de países, que incluso hablan de un sistema subyacente de control de ciertos jóvenes. Asimismo, la población recluida en Brasil es enorme, sólo comparable a otros países con sistemas carcelarios severos. México tiene una tasa cinco veces menor a Uruguay, tres veces menor a Brasil y de la mitad del promedio.

Cuadro 1 - Adolescentes procesados en 11 sistemas de justicia penal especializada Tasa por cada 100 mil adolescentes. c. 2015 (orden descendiente por tasa)

\begin{tabular}{|l|l|l|l|l|}
\hline \multicolumn{1}{|c|}{ País } & $\begin{array}{c}\text { Adolescentes } \\
\text { privados de la } \\
\text { libertad }\end{array}$ & $\begin{array}{c}\text { Total de } \\
\text { adolescentes } \\
\text { intervenidos } \\
\text { (incl. Medidas } \\
\text { en libertad) }\end{array}$ & $\begin{array}{c}\text { Tasa de } \\
\text { adolescentes en } \\
\text { el sistema }\end{array}$ & $\begin{array}{c}\text { Tasa de } \\
\text { adolescentes } \\
\text { privados de } \\
\text { libertad }\end{array}$ \\
\hline Uruguay & 473 & 753 & 83.6 & 52.5 \\
\hline Perú & 3,785 & 6,651 & 63.5 & 36.1 \\
\hline Brasil & 20,532 & 87,577 & 149.9 & 35.1 \\
\hline Chile & 1,537 & 13,461 & 297 & 33.9 \\
\hline Colombia & 3,539 & 12,654 & 79 & 22.1 \\
\hline Paraguay & 387 & n.d. & n.d. & 14.8 \\
\hline Argentina & 1,477 & 4,392 & 36.4 & 12.2 \\
\hline Ecuador & 625 & 761 & 13.6 & 11.2 \\
\hline México & 4,365 & 8,922 & 21.3 & 10.4 \\
\hline Bolivia & 269 & 365 & 8.3 & 6.1 \\
\hline Costa Rica & 40 & 761 & 54.5 & 2.9 \\
\hline Promedio & 3,366 & 13,630 & $\mathbf{8 0 . 7 1}$ & $\mathbf{2 1 . 5 7}$ \\
\hline $\begin{array}{l}\text { Desviación } \\
\text { estándar }\end{array}$ & 5,626 & 25,097 & 82 & 15 \\
\hline
\end{tabular}

Fuente: Tomado de Vernazza (2016, p. 2). 
Las implicaciones para las poblaciones juveniles son importantes. Con un promedio de población juvenil de 14 millones por país, las tasas indican que la incidencia de jóvenes procesados por infracciones son bajas (ver cuadro 1: 80.71 para todos los procesados y 21.57 para los privados de libertad), comparada con las de adultos, que para esos años era de 242 personas en prisión por cada 100 mil habitantes (World Prison Brief, 2021 y Vernazza, 2016: 2). Esto es una proporción de 11.22. No obstante, el promedio de las proporciones de menores encarcelados en los países del cuadro 1 es de $42 \%$ respecto del total de adolescentes procesados. Además, Uruguay y Brasil tienen tasas por encima del promedio y éste último tiene una enorme cantidad de adolescentes recluidos. Esto probablemente se debe a que las leyes y los sistemas judiciales clasifican las conductas de estos jóvenes como graves, lo cual sustenta una mayor propensión de los jueces a imponerles medidas de encierro.

La proporción entre hombres y mujeres es prácticamente de 1 a 10. La mayoría ha sido procesada por robo o delitos patrimoniales, luego por homicidio y por tráfico de drogas. Y estos son los principales delitos que justifican el encierro (cuadro 2).

Cuadro 2 - Adolescentes procesados por tres sistemas de justicia penal adolescente: Brasil, México y Uruguay y delito más frecuente

\begin{tabular}{|l|l|l|l|}
\hline \multicolumn{1}{|c|}{ País } & \multicolumn{1}{c|}{ Masculino } & \multicolumn{1}{c|}{ Femenino } & Tipo de delito que más frecuente \\
\hline Brasil (2020) & $95.3 \%$ & $4.7 \%$ & Robo y tráfico de drogas \\
\hline México $(2017)$ & $92.17 \%$ & $7.83 \%$ & Robo y homicidio \\
\hline Uruguay $(2019)$ & $93 \%$ & $7 \%$ & Robo y rapiña \\
\hline
\end{tabular}

Fuente: Datos tomados de los capítulos 1,3 y 4 de Alvarado y Tenenbaum (2022).

\section{Un solo perfil de los adolescentes procesados}

El grupo que estudiamos está compuesto por adolescentes que han sido procesados con base en la Ley Nacional del Sistema Integral de Justicia Penal para Adolescentes (SIJPA) (Cámara de Diputados, 2016). Es un subconjunto 
de un grupo de jóvenes infractores de la ley que incluye personas hasta los 24 años, pero cuyo tamaño es desconocido hasta ahora, dado que no existen estimaciones completas de los presuntos responsables y porque además hay una enorme cifra negra de delitos en la que las víctimas señalan que sus agresores fueron adolescentes (ver la ENVIPE o la ENSU). ${ }^{2}$ Hay una cifra aún mayor y desconocida de jóvenes que han sido detenidos por las policías y que aún en las fiscalías han negociado su liberación. Los datos del SIJPA ofrecen registros sobre los cuales puede establecerse el perfil que nos interesa. El sistema estadístico público y las instituciones penales utilizan un conjunto de categorías que encuadran a los adolescentes "institucionalizados" dentro de un marco que ellos definen como problemático, lo cual debe analizarse críticamente y desentrañar los supuestos que incriminan y etiquetan a los jóvenes. Las instituciones policiales y las penales construyen al joven delincuente en sus marcos normativos y de acción, así como en sus actividades rutinarias.

\section{La población en proceso penal}

En México, en el año 2019 había 28 mil 371 adolescentes (personas entre 12 y menos de 18 años) registrados en las averiguaciones previas, investigaciones y carpetas de investigación abiertas por el Ministerio Público como probables responsables de cometer diversos delitos. ${ }^{3}$ El $74.5 \%$ de ellos eran hombres y $14.8 \%$ mujeres (el resto no estaba identificado). La proporción de menores imputados era de 1.67 por cada 100 adultos. Respecto de los delitos, ese año se registraron 1 millón 950 mil 717

\footnotetext{
${ }^{2}$ La ENVIPE es la Encuesta nacional de victimización y percepción sobre seguridad pública. La ENSU es la Encuesta nacional sobre seguridad pública urbana. Ambas son levantadas regularmente por el Instituto Nacional de Estadística y Geografía de México.

${ }^{3}$ Datos del Censo Nacional de Procuración de Justicia Estatal 2020 registrado por el Instituto Nacional de Estadística y Geografía, INEGI. Disponible en: https://www.inegi.org.mx/ programas/cnpje/2020/.
} 
(presuntos) delitos de los cuales $1.21 \%$ correspondían al sistema de adolescentes. La proporción de población registrada es pequeña frente al universo de presuntos agresores; así también su participación en actos delictivos. La gran mayoría de los jóvenes (imputados o procesados) fueron detectados por el sistema de justicia por primera vez y hay muy pocos reincidentes.

\section{Perfiles de la Encuesta Nacional de Adolescentes en el Sistema de Justicia Penal}

Para conocer mejor los perfiles de los sujetos procesados utilizamos la Encuesta Nacional de Adolescentes en el Sistema de Justicia Penal (ENASJUP) de 2017, ${ }^{4}$ que cubrió una muestra de 3 mil 308 adolescentes residentes en 53 centros de internamiento en las 32 entidades federativas de México. Es una muestra de un total de 6 mil 891 adolescentes registrados en el sistema, a quienes se les dictó o estaba por dictar alguna medida judicial. El 92.2\% de ellos eran hombres y $7.8 \%$ mujeres; al $23.4 \%$ se les había aplicado medidas de encierro dentro del sistema y $76.6 \%$ contaba con medidas de externación (por lo que no conocemos la mayoría de sus datos) (INEGI, 2018).

Además, durante 2019, ingresaron a esos centros 4 mil 119 personas, que se sumaron a las que ya estaban dentro del sistema. Estas cifras son muy pequeñas si las comparamos con los datos de población penitenciaria, pero no por esto dejan de ser importantes, porque la proporción era de un 1.6 menores por cada 100 presos adultos. ${ }^{5}$ Esto representa una tercera parte de todos los adolescentes que están imputados en los registros de las fiscalías, lo cual proporciona la impresión de que la policía es más efectiva en detener a los menores que a los adultos.

${ }^{4}$ Encuesta Nacional de Adolescentes en el Sistema de Justicia Penal (ENASJUP). Tabulados, INEGI, 2017. Disponible en: https://www.inegi.org.mx/programas/enasjup/2017/\#Tabulados ${ }^{5}$ Datos del Censo de Nacional de Procuración de Justicia y del Censo Nacional de Gobierno, Seguridad Pública y Sistema Penitenciario Estatales (CNGSPSP) (INEGI, 2020). 
Respecto a su distribución en grupos de edad, 59.4\% tenía entre 18 y 22 años; le sigue el grupo entre 16 y 17 años con 25.5\%; y un grupo de jóvenes de 14 a 15 años con 9.5\% (INEGI, 2018). Hay un pequeño grupo de adolescentes entre 12 y 13 años.

Gráfica 1 - Distribución por grupos de edad de la población en centros de tratamiento especializado del SIJPA, 2017

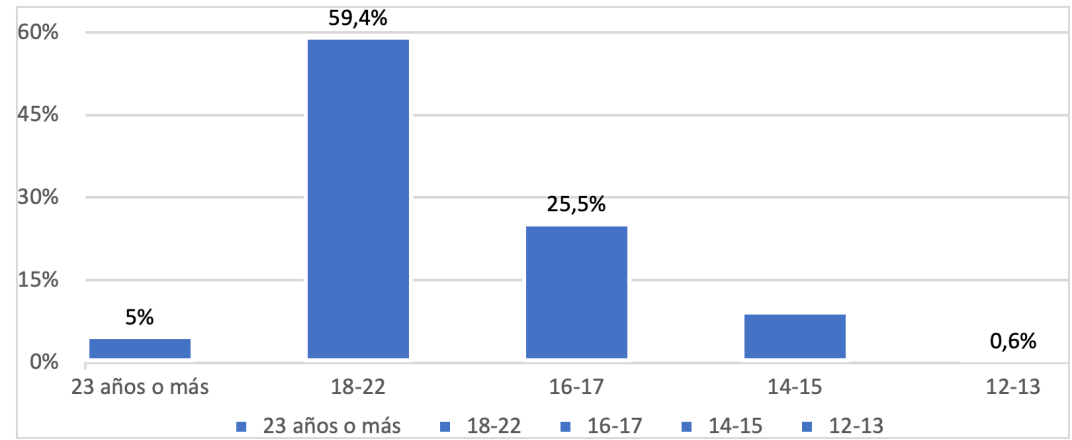

Fuente: Datos tomados de INEGI, 2018.

En cuanto a la distribución geográfica, el estado con más adolescentes en el sistema de justicia penal es Jalisco, con 2,102 individuos (equivalente a $30.51 \%$ del total); seguido por Tlaxcala con $20.76 \%$, Sonora con 11.49\%, Chihuahua con $9.8 \%$ y Tamaulipas con $4.47 \%$. Estas cinco entidades suman $77 \%$ de los adolescentes procesados, no obstante, no son las más pobladas (por lo que tienen tasas de encarcelamiento más altas que el promedio, de 10.1) ni las que tienen mayor frecuencia de actos delictivos. 


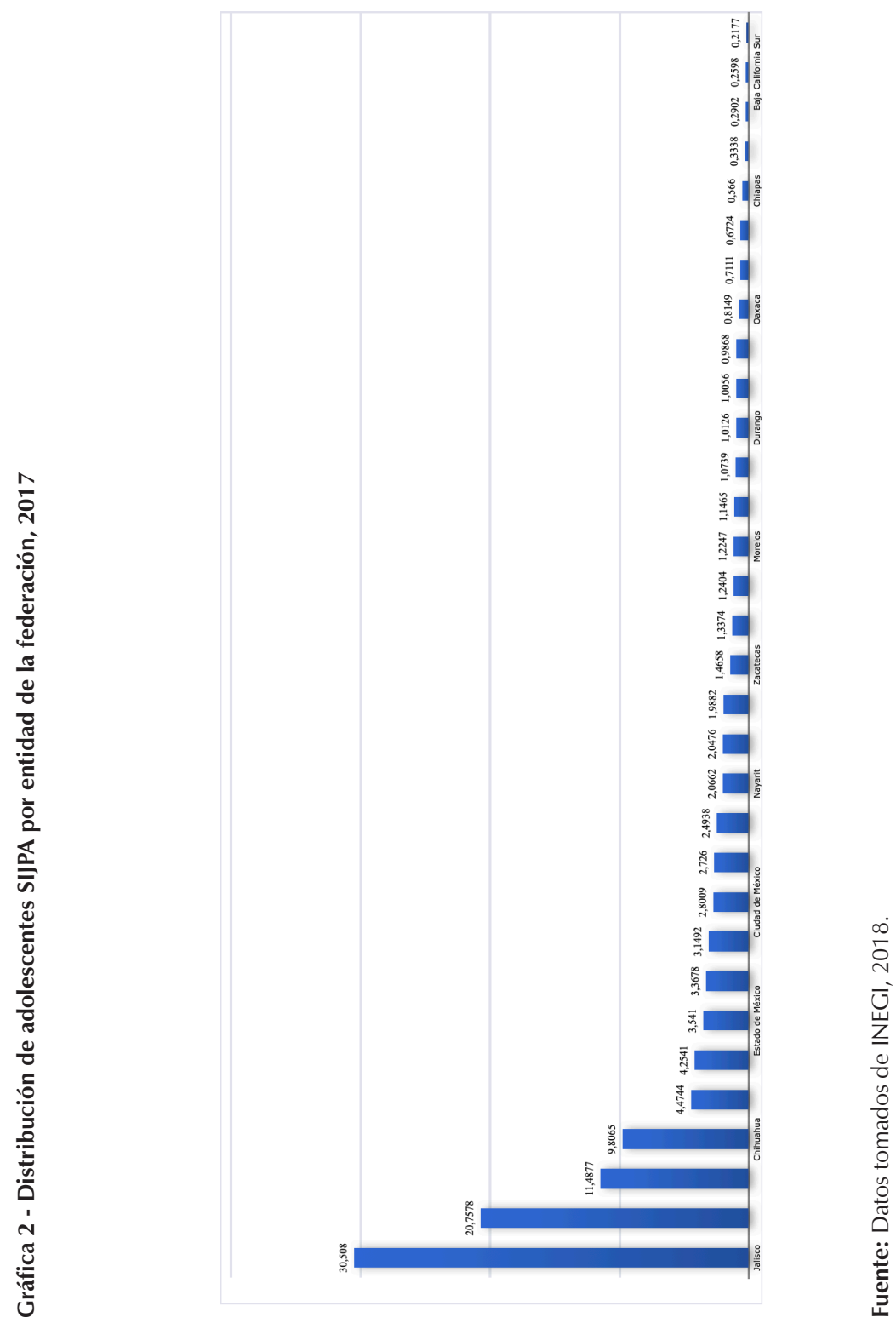

Sociologias, Porto Alegre, ano 23, n. 58, set-dez 2021, p. 50-78. 


\section{Perfil sociodemográfico}

La gran mayoría de los adolescentes residentes en las instituciones del SIJPA sabía leer y escribir (95.3\%); sin embargo, 75.9\% sólo contaba con educación básica, mientras que 22.1\% cursaba educación media y $1.1 \%$ superior (INEGI, 2018). De hecho, la gran mayoría se encontraba estudiando al momento de la detención, aun cuando tuviera un claro rezago educativo (2 mil 681 adolescentes procesados estaban fuera de la escuela) (CNDH, 2019, p. 369).

De ellos, $71.2 \%$ era soltero/a, $21.6 \%$ vivía en pareja y el $7.3 \%$ estuvo casado/a alguna vez. Un mil 505 afirmaron tener al menos un hijo, es decir 21.8\% (uno de cada cinco), lo cual podría implicar algunas responsabilidades familiares y dinámicas de la relación de pareja a edades tempranas - son padres adolescentes. Más 23.5\% contaba con dependientes económicos antes de su detención. Para las mujeres, esto significaba embarazos adolescentes $-46.7 \%$ de ellas estuvo embarazada alguna vez en su vida y $18.6 \%$ ha tenido al menos un aborto (INEGI, 2018). La relación afectiva y las dinámicas de pareja aparecieron en la adolescencia temprana con eventos complejos.

La gran mayoría de ellos trabajaba desde la infancia. El 5.6\% de ellos tuvo su primer trabajo antes de cumplir los 10 años; 68\% obtuvo su primer empleo entre los 10 y 15 años y $24.8 \%$ entre 16 y18 años. El 94.3\% de los adolescentes declaró haber trabajado alguna vez y solo $5.7 \%$ de ellos no lo había hecho antes de entrar al sistema. De los jóvenes, 57\% declaró que requería trabajar para contribuir al ingreso de sus hogares y $53.7 \%$ tenía la necesidad de trabajar los siete días de la semana. El 91.7\% afirmó que contaba con suficiencia alimentaria; 85\% tenía acceso a algún tipo de régimen de salud, contaba con recursos para pagarse ropa, calzado, la renta de la vivienda y $25 \%$ tenía deudas. Al momento de ser detenido el $67.3 \%$ de los adolescentes contaba con empleo, esto es, lo combinaba o alternaba con las actividades infractoras. 
Cuadro 3. Situación de empleo de los adolescentes en el SIJPA la semana previa de su detención

\begin{tabular}{|c|c|c|c|}
\hline Ocupación & $\%$ & Motivo por el que no trabajaba & $\%$ \\
\hline $\begin{array}{l}\text { Trabajadores en actividades } \\
\text { elementales y de aрoуo }\end{array}$ & 40.3 & Estaba estudiando & 50.6 \\
\hline Trabajadores artesanales & 22.1 & $\begin{array}{l}\text { No quería, no tenía interés o } \\
\text { necesidad }\end{array}$ & 17.3 \\
\hline $\begin{array}{l}\text { Comerciantes, empleados en } \\
\text { ventas y agentes de ventas }\end{array}$ & 10.9 & Me drogaba y tomaba & 6.2 \\
\hline $\begin{array}{l}\text { Trabajadores en servicios } \\
\text { personales y vigilancia }\end{array}$ & 8.2 & No había oportunidades de trabajo & 5.0 \\
\hline $\begin{array}{l}\text { Trabajadores en actividades } \\
\text { agrícolas }\end{array}$ & 7 & $\begin{array}{l}\text { Me dedicaba a las labores del } \\
\text { hogar y/o cuidar a un familiar }\end{array}$ & 5.0 \\
\hline Profesionistas y técnicos & 4.2 & $\begin{array}{l}\text { Renuncié, cerró la empresa o } \\
\text { estaba buscando trabajo }\end{array}$ & 4.0 \\
\hline $\begin{array}{l}\text { Operadores de maquinaria } \\
\text { industrial, ensambladores, } \\
\text { conductores de transporte }\end{array}$ & 4.1 & No tenía permiso, edad o tiempo & 3.2 \\
\hline $\begin{array}{l}\text { Trabajadores auxiliares en } \\
\text { actividades administrativas }\end{array}$ & 1.7 & $\begin{array}{l}\text { No me aceptaron, no cubría el } \\
\text { perfil, me corrieron }\end{array}$ & 2.1 \\
\hline Funcionarios, directores y jefes & 0.2 & $\begin{array}{l}\text { Por condiciones laborales (poca } \\
\text { paga, jornada laboral extensa, } \\
\text { lejanía etc.) }\end{array}$ & 0.9 \\
\hline \multirow[t]{2}{*}{ No especificado } & 1.3 & Por cuestiones de salud & 0.8 \\
\hline & & Otro & 3.7 \\
\hline
\end{tabular}

Fuente: Elaboración propia con datos de INEGI, 2018.

Buena parte de los adolescentes que cometieron delitos estaba inserta en el sistema educativo y en el mercado de trabajo antes de ser detenida. Por supuesto, la escolarización no implica necesariamente atención o 
calidad escolar. Tampoco nos habla de los niveles de logro o si es atractiva para ellos. Lo mismo podría decirse del mercado de trabajo, dado que las ocupaciones son precarias, inestables, mal pagadas, sin protección ni beneficios.

Los trabajadores tenían empleos marginales y los alternaban y combinaban con actividades delictivas. La mayoría de los procesados contaba con baja capacitación laboral y con contratos y remuneracion precaria, pero hay un grupo de artesanos, técnicos y profesionistas con mayor capital humano. El 8\% hacía labores de vigilancia. Pero hay que recalcar que los detenidos o estudiaban o trabajaban.

A esto hay que sumar el contexto barrial y familiar - varios de los adolescentes tenían conflictos familiares; y los barrios en donde vivían tenían carencias importantes y presencia de varias formas de violencia. ${ }^{6}$

\section{Condición de los hogares de origen y dinámica de las relaciones en la familia}

De los adolescentes en el SIJPA, 32.8\% estaba bajo la tutela del padre y la madre, mientras que el $39.8 \%$ de ellos estaba bajo la tutela de la madre. Ahora bien, $39.9 \%$ de los adolescentes ya había abandonado su hogar al menos una ocasión; 3\% salió antes de los 10 años y 65\% de ellos lo hizo cuando tenía entre 10 y 15 años; 30.8\% lo hizo a los 16 años o después. Al salir, una tercera parte se fue a vivir a casa de un amigo; $30 \%$ con otro familiar; $17 \%$ con el novio/a, $11 \%$ se fue a vivir solo, mientras que $6.1 \%$ vivió en situación de calle. Los encuestados tuvieron muchas dificultades desde la niñez y enfrentaban algún tipo de conflicto con los padres o la familia. Además, concentraban desventajas educativas y económicas y situaciones de maltrato familiar, social y gubernamental desde la infancia. No son jóvenes de clase media, sino de una parte de una clase proletaria

\footnotetext{
${ }^{6}$ Varios autores han estudiado el entorno de barrios violentos, la presencia de bandas armadas que controlan varias actividades y servicios legales e ilegales en estos territorios; algunas con capacidad de ejercer coerción a la población (Véase Alvarado, 2014).
} 
que vive en condiciones de marginación y privación relativa alta. Al mismo tiempo, son afectados por la estructura de oportunidades muy limitada por su origen familiar, por su residencia, escolaridad, y otras condiciones de uniones tempranas y embarazos adolescentes.

\section{Gráfica 3 - Condición de tutela del menor en el SIJPA}

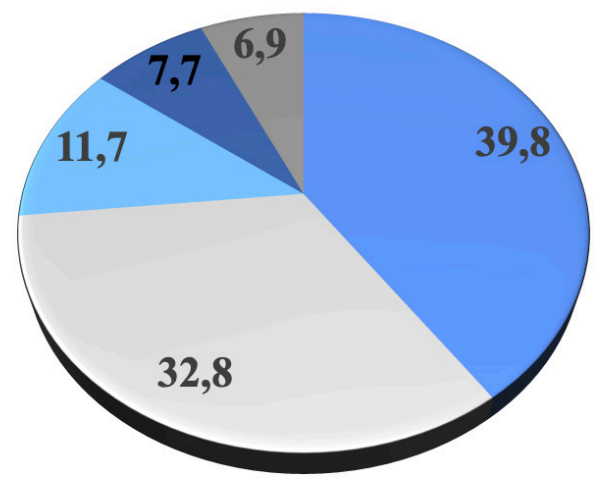

\section{Madre \\ Padre y madre \\ Otro adulto \\ Nadie \\ Padre}

Fuente: Elaboración propia con datos tomados de INEGI, 2018.

\section{Perfil de los delitos}

¿Cuáles son las principales infracciones que cometieron los adolescentes? Hay seis grupos de infracciones predominantes: fueron detenidos por robo, ${ }^{7}$ homicidio, violación y otros delitos sexuales, portación ilegal de armas, posesión o comercio de drogas y secuestro (cuadro 4). El delito más común era el robo, que asciende a $37 \%$ del total y la edad promedio de los jóvenes procesados era los 15 años (pero sólo a 7.8\% le fue dictada una sanción por este delito).

${ }_{7}^{7}$ Dentro de la categoría de robo están aquellos a negocios, a transeúntes y de autos. 
Cuadro 4. Infracciones de las y los adolescentes institucionalizados

\begin{tabular}{|c|c|c|}
\hline Delito equiparable & $\%$ & Edad promedio \\
\hline Robos & 33.9 & 15 \\
\hline Homicidio & 7.9 & 16 \\
\hline Violación sexual & 7.8 & 14 \\
\hline Posesión ilegal de drogas & 3.8 & 16 \\
\hline Lesiones & 3.8 & 15 \\
\hline Comercio ilegal de drogas & 3.0 & 15 \\
\hline Secuestro & 2.3 & 16 \\
\hline Portación ilegal de armas & 1.9 & 16 \\
\hline Delitos sexuales & 1.9 & 14 \\
\hline Violencia familiar & 0.9 & 16 \\
\hline Extorsión & 0.6 & 16 \\
\hline Secuestro exprés & 0.6 & 17 \\
\hline Daño a la propiedad & 0.5 & 14 \\
\hline Delincuencia organizada & 0.2 & 15 \\
\hline Privación de la libertad & 0.1 & 15 \\
\hline Amenazas & 0.1 & 14 \\
\hline Abuso de confianza & 0.0 & 15 \\
\hline Fraude & 0.0 & 15 \\
\hline Otro delito & 16.5 & 15 \\
\hline Delitos en grado de tentativas & 14.4 & 15 \\
\hline Riña & 14.1 & 13 \\
\hline $\begin{array}{l}\text { Conductas delictivas de las que se desconoce el } \\
\text { resultado }\end{array}$ & 11.8 & 16 \\
\hline Delitos contra la salud & 8.0 & 15 \\
\hline Pandillerismo & 6.1 & 15 \\
\hline Pinta en propiedad ajena (graffiti) & 5.6 & 14 \\
\hline Otras conductas no delictivas & 24.1 & 16 \\
\hline
\end{tabular}

Fuente: Datos tomados de INEGI, 2018. 
La mayoría de los delitos por los que fueron procesados son patrimoniales (el robo de vehículo junto con robo a transeúnte y a pequeños negocios representa buena parte de ellos), siguiendo el homicidio, pero en menor proporción, los delitos sexuales, delitos relacionados con drogas ilícitas o contra la salud, delitos contra la seguridad pública y muchos otros delitos que, en realidad, son conductas asociadas con comportamientos adolescentes, como el grafiti, las riñas y el pandillerismo, pero que están registrados en algunos códigos penales estatales. Pero cabe hacer notar que muchas detenciones fueron por faltas administrativas o cívicas no penales, pero luego se agregaron otros eventos.

El 50.5\% fue acusado por delitos del fuero común, mientras que $15.9 \%$ por delitos del fuero federal (INEGI, 2018), lo cual puede haber implicado una detención con autoridades federales o militares, así como también la comisión de delitos del código penal federal. De acuerdo con la Comisión Nacional de los Derechos Humanos, CNDH, la ENASJUP reporta medidas de sanción dictadas por participar en actos propios de delincuencia organizada, aunque solo representan el 2.5 por ciento del total. Estas conductas son: secuestro (34.9), acopio ilegal de armas (23.6), venta de droga (17.4), homicidio (17.2), robo (14.7) y extorsión (1.4) (CNDH, 2019, p. 369).

Hay, sin embargo, muchos problemas adicionales a la delincuencia "común". En el país hay numerosos conflictos y formas de violencia extrema, como los asesinatos, los secuestros, el tráfico de personas, los desplazamientos forzados, las desapariciones (CNDH, 2019, p. 363). Todo esto habla de que existen varios conflictos armados en el país - por los enfrentamientos entre organizaciones criminales y de éstas contra el gobierno - en los que los jóvenes son más bien víctimas. Así que, además de las tendencias delictivas que se reflejan en la estadística publicada, hay que agregar otros temas a la conducta desviada, como la participación en pandillas violentas o el involucramiento o el reclutamiento forzado en organizaciones criminales, que utilizan menores de edad para labores de 
apoyo que pueden ir desde servir de informantes, pasando por otras formas de involucramiento ilícito forzado como cuidadores de víctimas, sicarios (Encinas Garza, 2016), vendedores o distribuidores de mercancías ilícitas etc. Azaola (2020, p. 133) define tres grupos.

1. Un primer grupo de $35 \%$ que dijo ser parte de un grupo de delincuencia organizada.

2. Un segundo grupo que dijo haber cometido el delito como parte de alguna pandilla o banda de su colonia (27\%).

3. Un grupo que delinquió solo o con amigos (38\%).

Esta clasificación y los perfiles de los jóvenes coincide con nuestros datos. En entrevistas propias realizadas durante un estudio en la Ciudad de México, en 2018, se encontraron algunos casos donde ingresan jóvenes pertenecientes a organizaciones criminales, por lo que las autoridades consideraron hacer una separación espacial de estos con el resto de los detenidos. Entonces, se hace una clasificación y separación no necesariamente a partir de las edades, sino del tipo de personalidad de las y los chicos; su pertenencia a un grupo o banda delictiva; su capacidad para socializar con otros compañeros. Varios trabajadores de los centros de tratamiento se sorprenden cuando encuentran adolescentes muy jóvenes, pero con una fuerte personalidad y capacidad de dominación y coerción hacia sus compañeros más grandes. Pero otros factores de personalidad asociados con las conductas agresoras deben integrarse en los estudios y los supuestos psicosociales.

En muchas ocasiones los adolescentes no cuentan con opciones para decidir libremente; su capacidad de agencia está muy constreñida cuando viven en barrios y ciudades afectadas por altas tasas de violencia, por las condiciones familiares mencionadas, por oportunidades de educación y empleo precarias y en algunas ocasiones son capturados por organizaciones criminales y son víctimas de los mismos crímenes. 


\section{Narrativas sobre las experiencias delictivas}

Estos jóvenes comparten muchos valores culturales, de consumo, dominantes en la sociedad, pero la estructura de oportunidades no les ha permitido optar por una conducta "normal" para materializar sus deseos. $\mathrm{Y}$ en algunas ocasiones, al envolverse en actividades de pandillas o de organizaciones criminales sus aspiraciones cambian.

Los perfiles de los adolescentes coinciden en la preferencia por los actos delictivos como algo excitante, pero no necesariamente les desagrada el trabajo; algunos trabajan, pero muchos desprecian las características precarias y los salarios bajos de los empleos a los que tienen acceso. La mayoría presenta rasgos de una masculinidad violenta. Sin embargo, en las entrevistas que hemos realizado y en otros trabajos, las aspiraciones de los jóvenes se dividen en dos grupos, uno que desearía una vida como trabajador reconocido (incluso, algunos mencionaron que les gustaría trabajar en el ejército (Azaola, 2020), en donde tenga reconocimiento social y familiar, y otro que tiene la ilusión de ser como algunos criminales de organizaciones mexicanas. Precisamente, en su estudio de jóvenes detenidos en México, Azaola examina las motivaciones de los entrevistados que forman parte de los grupos de delincuencia organizada y encuentra que, entre un grupo, "existe el deseo de imitar un estilo de vida que los adolescentes han podido observar en su entorno y que incluye armas, autos, alcohol, drogas, sensación de poder y otros lujos" (Azaola, 2020, p. 134). Incluso aspiran a formar parte de esos grupos - según Azaola (p. 134), "que les brinda la sensación de pertenencia, de protección, de solidaridad, equivalentes o sustitutas a las de una familia").

Los estudios de adolescentes recluidos muestran que sí tienen conciencia de sus actos, no niegan su responsabilidad, pero los neutralizan y justifican - como proponen Matza y Sykes (1961). En la encuesta, la mitad de los adolescentes se declaró culpable ante el Ministerio Público. De ellos, 74.6\% 
reconoció los hechos. En el estudio de Azaola, los entrevistados construyen narrativas en las que, si bien no niegan el daño, disminuyen el valor de la víctima, ya sea porque realizaba conductas indebidas o porque se trataba de un "enemigo", o afirman que solo obedecen órdenes (Azaola, 2020).

Hay un considerable uso de armas de fuego en los delitos (Concha et al., 2020). En varias entrevistas, en un estudio de violencia juvenil en América Latina, buena parte de los entrevistados conocían las armas y sabían que podían obtenerlas con facilidad en sus barrios (Alvarado, 2014). El 70\% de los entrevistados utilizó un arma en la comisión del delito por el que lo procesaron (51\% pistolas, 29\% armas punzocortantes, 10\% armas de fuego de alto poder y $10 \%$ palos, piedras, botellas). Un aspecto complementario es el imaginario social que asocia a los adultos y jóvenes que cometen delitos con figuras como La Santa Muerte y/o Malverde (o San Judas Tadeo, una tradición más vieja) (Yllescas, 2019). Mas en el caso de la Ciudad de México, del total de 210 jóvenes internados en 2019, 18.5\% no profesaba ninguna religión; $6.2 \%$ declaró pertenecer a "otra"; $5.7 \%$ es cristiano; $4.2 \%$ es santero; pero 3.8\% cree en la Santa Muerte $^{8}$ y $1.4 \%$ es católico.

\section{Primodelicuentes, amateurs y delicuentes habituales}

A partir de la presentación de los datos, encontramos tres tipos de jóvenes procesados-capturados por el sistema; un primer tipo es el que es capturado a edad "temprana" desde los 12 años pero sobre todo entre los 14 y 15 años, que no tiene antecedentes - son primodelicuentes (ver la gráfica 1); un segundo dentro del grupo etario en que se concentra la población entre 16 y 17 años, que podría considerarse el delincuente amateur; y un tercer grupo desde los 18 años en el cual se concentra la mayoría de los institucionalizados por el régimen. Este último grupo, con

${ }^{8}$ En una visita a la Comunidad de Mujeres en la Ciudad de México en 2018 observamos dibujos de La Santa Muerte en dos de los dormitorios (González, 2020). 
trayectoria delictiva, incluye a los reincidentes y registra muchos otros actos delictivos previos por los que no fueron procesados (ENSAJUP*). Luego, podría hacerse una clasificación más fina de las personas que cuentan con experiencia delictiva - de acuerdo con la forma en que cometieron los delitos, así como la probable pertenencia a una organización criminal - ligada a las narrativas de sus actos y a su perfil aspiracional, las que producirían dos subgrupos: uno que tiene narrativas de haber cometido varios delitos antes de ser detenidos y procesado y otro grupo que, además, se caracteriza por tener aspiraciones ligadas a personalidades criminales "populares". El perfil sociodemográfico y familiar es muy similar entre los grupos.

El sistema ha procesado pocos reincidentes pero gran parte de los recluidos contaba con eventos delictivos previos. En la ENASJUP, 9.7\% contaba ya con un proceso previo y $5 \%$ de ellos ya había estado en una comunidad de internamiento. Además, 19.1\% (uno de cada cinco) declaró haber realizado otra actividad ilícita, sin haber sido aprehendido (y entre ellos $60 \%$ declaró haberlo hecho entre una y cinco veces; $13.3 \%$ entre 6 y 10 veces y $16.7 \%$ más de diez veces); $25.2 \%$ de los adolescentes fue capturado en el acto (infraganti); $33.3 \%$ fue detenido inmediatamente después de realizar la conducta que se le imputaba; 22.5\% tenía orden de detención. Hay un grupo con el perfil de una carrera criminal temprana.

\section{Discusión}

Tanto en México como en América Latina se ha documentado que el fenómeno de la judicialización de los jóvenes tiene una relación con la desigualdad - los menores procesados no han contado con los recursos suficientes para resolver necesidades y derechos básicos, por lo que probablemente buscan una alternativa de subsistencia con las actividades ilícitas (Melo, 2011; Jones; Rodgers, 2009; Rodríguez, 2004). Pero, por otro lado, se entrecruza un fenómeno propio de las tareas judiciales que tiene que ver con la persecución o policiamiento (Kessler; Dimarco, 2013) de 
los estratos más vulnerables de la sociedad que son detenidos. En buena parte de los casos se trata más bien de detenciones arbitrarias, con uso excesivo de la fuerza física y son remitidos a los fiscales porque no pudieron negociar su liberación (en la ENASJUP, los jóvenes reportaron 107 casos de corrupción policial y 88 del MP). ${ }^{9}$ Estas prácticas dejan fuera de la justicia penal a quienes poseen más y mejor acceso a otro tipo de estrategias que permiten evitar dicho proceso y alcanzar un acuerdo fuera de los tribunales. Las dinámicas delictivas pueden ser las mismas, pero los recursos de capital económico, político y las redes de apoyo que neutralizan la acción policial marcan la diferencia.

El régimen policial establece mecanismos de control de esta población y privilegia la detención después de un proceso ilegal y extorsivo contra los adolescentes que se encuentran en los espacios de interacción en donde se concentran características de desorganización social. Capturan a quienes, por sus mismas condiciones de escasez, no siempre cuentan con la posibilidad de acceder a una defensa adecuada, redes o estrategias que les permitan evitar la judicialización. Esta perspectiva es compartida por las autoridades del sistema de adolescentes.

Así que, además del problema de adolescentes infractores con carreras criminales tempranas, hemos puesto en evidencia el otro gran problema, de un sistema policial y judicial concentrado en perseguir, etiquetar y procesar injusta e ilegalmente los jóvenes más vulnerables, dejando a otros grupos sociales fuera del radar de la justicia penal.

En ese sentido, se ha difundido la idea de que son los jóvenes (y los adultos) más pobres quienes cometen buena parte de los delitos, con estos registros que acompañan la construcción de una imagen de peligrosidad (Canales, 2008; Jones; Rodgers, 2009; Castillo, 2004). El fenómeno de la delincuencia juvenil es una construcción pública de las instituciones policiales y penales del estado, que etiqueta e impone un control social a ciertas poblaciones, a través de su criminalización-judicialización y castigo. Sin embargo, las narrativas de los propios procesados muestran que hay una heterogeneidad en los valores y aspiraciones; la gran mayoría de los procesados reconoce los actos, pero

${ }^{9}$ Ver también: Vega (2014), González y Vera (2011). 
aspira a alternativas de vida diferentes. Es cierto, como proponen Matza y Sykes (1961) que la delincuencia juvenil puede ser producto de distorsiones a la personalidad, conflictos emocionales que responden a un ambiente social en forma desviada (en contraste con aquellos que responden obedeciendo las leyes y las normas culturales dominantes). No obstante, hay que destacar que, si bien hay un etiquetamiento de los individuos que producen estos actos desviados y una recriminación predominante de esta conducta a las clases bajas, en realidad, buena parte de la población de todos los estratos sociales y culturales comparte esa ambigüedad de valores, que combinan un bajo respeto a la ley con aspiraciones personales hacia un mejor futuro.

El sistema procesa un solo perfil de ofensor, que concentra características sociodemográficas, sociales, personales y territoriales de extrema precariedad, abandono y vulnerabilidad. De la condición familiar, social y urbana de los jóvenes resultaron los elementos que los definen como fuera de la ley. Esto también se refleja en las edades en las que los adolescentes reportan haber comenzado a cometer delitos. Comparten rasgos de infancia con privaciones importantes, con relaciones familiares conflictivas, sobre todo con el padre. La madre juega un papel importante. Trabajan por necesidad personal y familiar y, en ocasiones, esto desplaza la escuela. Combinan estas actividades con la ilícitas, lo que indica una mezcla de necesidad y oportunidad. Además, en muchos casos, hay una clara presencia de alcohol, drogas, violencia en sus familias y barrios y de antecedentes penales de algunos miembros de la familia o de amigos. Junto con esto, el trabajo policial tan focalizado contribuye a etiquetar estos individuos en un grupo social peligroso. Pero la combinación de todos estos factores no necesariamente coincide con muchas clasificaciones del delito juvenil. La conducta delictiva juvenil no sólo puede explicarse en función de factores como la propensión al riesgo, o la exposición a valores culturales y oportunidades en conflicto, sino a un conjunto acumulado de privaciones que se suma a una forma de trabajo de las instituciones de control social. El joven delinque no porque tenga valores sociales diferentes, sino porque considera que es la mejor forma de acceder a los valores y a los productos deseados, o porque la estructura de oportunidades no le ha permitido elegir libremente qué conducta seguir. También mostramos varios casos donde cometer actividades delictivas es forzoso. 


\section{Consecuencias para los adolescentes}

En la vida de estos jóvenes, plena de privaciones y exclusión, el haber sido institucionalizados es una estación que puede otorgarles un descanso entre varias rutas: la primera, la de la delincuencia como carrera; la segunda, una pausa de su curso de vida en un entorno muy conflictivo. La mayoría de las y los jóvenes formará un hogar y una familia y encontrará un nicho de trabajo que eventualmente lo lleve a desistir de esa carrera. Continuarán una vida precaria, seguirán participando en el mundo ilegal, pero no como delincuentes de carrera.

Lo trascendente es que, con la captura, comienza un proceso de institucionalización que formalmente les reconoce derechos y a su vez los etiqueta, los convierte en sujetos y objetos del Estado, que están en riesgo de cometer delitos - sus pacientes. El sistema policial y de justicia penal ha creado un tipo de delincuente que no será transformado ni rehabilitado. Seguirá siendo objeto de abusos y reproducirá las carencias y la situación de precariedad de donde provienen.

Arturo Alvarado Mendoza es Doctor en Ciencia Social con especialidad en Sociología y Director del Colegio de México.

$\bowtie$ alvarado@colmex.mx

\section{Referencias}

1. ALVARADO, Arturo; TENENBAUM, Gabriel (eds.) Los desafíos de la justicia para adolescentes en América Latina. Ciudad de México: El Colegio de México, en prensa.

2. ALVARADO, Arturo (ed.). Violencia juvenil y acceso a la justicia en América Latina. Ciudad de México: El Colegio de México, 2014. (Tomos I y II)

3. AZAOLA, Elena. Nuestros niños sicarios. México: Fontamara, 2020.

4. BECKER, Howard. Outsiders-defining deviance. Londres: Free Press of Glencoe, 1963.

5. CÁMARA DE DIPUTADOS. Ley Nacional del Sistema Integral de Justicia Penal para Adolescentes. Ciudad de México: Cámara de Diputados, 2016. Disponible en: http://www.diputados.gob.mx/LeyesBiblio/pdf/LNSIJPA.pdf 
6. CANALES, Manuel. Una aproximación a los factores que inciden en la comisión de delitos en adolescentes. Señales, n. 1, p. 49-72, 2008.

7. CASTILLO, Héctor. Pandillas, jóvenes y violencia. Desacatos Revista de Ciencias Sociales, n. 14, p. 105-126, 2004.

8. CNDH - Comisión Nacional de los Derechos Humanos. Informe especial de la Comisión Nacional de los Derechos Humanos sobre los Centros de Tratamiento Interno para los adolescentes que infringen la ley penal de la República Mexicana. CNDH, 2019. Disponible en: https://www.cndh.org.mx/sites/default/files/ documentos/2019-05/ADOLESCENTES-INFORME-ESPECIAL-2019.pdf

9. COHEN, Lawrence; FELSON, Marcus. Social change and crime rate trends: a routine activity approach. American Sociological Review, v. 44, n. 4, p. 588-608, 1979.

10. COHEN, Stanley. Folk devils and moral panics: the creation of the Mods and Rockers. Londres: MacGibbon \& Kee, 1972.

11. CONCHA-EASTMAN Alberto; MUÑOZ, Edgar; RENNÓ-SANTOS, Mateus. Homicides in Latin America and the Caribbean. In: BADA Xóchitl; RIVERASÁNCHEZ, Liliana. The Oxford Handbook of the Sociology of Latin America. Nueva York: Oxford University Press, 2020.

12. ENCINAS GARZA, José L. Jóvenes sicarios, la generación desechable: vivir rápido y morir joven. Ciencia UANL, v. 19, n. 80, p. 59-65, 2016.

13. GARLAND, David. A cultura do controle: crime e ordem social na sociedade contemporânea. Rio de Janeiro: Revan, 2008.

14. GONZÁLEZ, Paulina. La reproducción de la desigualdad para los adolescentes en el sistema de justicia penal con medida en internamiento de la Ciudad de México. Tesis (Maestría en Sociología Política) - Instituto de Investigaciones Dr. José María Luis Mora, México, 2020.

15. GONZÁLEZ, Violeta; VERA, Andrea. La violencia policial contra niños, niñas y adolescentes. Hacia un estado de derecho que ejerza efectivamente la protección contra toda forma de violencia. Asunción: CDIA; CODEHUPY, 2011.

16. INEGI - Instituto Nacional de Estadística y Geografía. Encuesta Nacional de Victimización y Percepción sobre Seguridad Pública (ENVIPE). Aguascalientes: INEGI, 2021

17. INEGI - Instituto Nacional de Estadística y Geografía. Encuesta Nacional de Seguridad Pública Urbana (ENSU). Aguascalientes: INEGI, 2021.

18. INEGI - Instituto Nacional de Estadística y Geografía. Censo de procuración de justicia. Aguascalientes: INEGI, 2020. Disponible en: https://www.inegi.org.mx/ contenidos/programas/cnpje/2020/doc/cnpje_2020_resultados.pdf

19. INEGI - Instituto Nacional de Estadística y Geografía. Resultados de la primera Encuesta Nacional de Adolescentes en el Sistema de Justicia Penal, 2017. Aguascalientes: INEGI, 2018. Disponible en: https://www.inegi.org.mx/ contenidos/saladeprensa/boletines/2018/EstSegPub/ENASJUP2017.pdf 
20. JONES, Gareth A.; RODGERS, Denis. Youth violence in Latin America: an overview and agenda for research. In: JONES, Gareth; RODGERS, Denis (eds.). Youth violence in Latin America. Gangs and juvenile justice in perspective. Nueva York: Palgrave Macmillan, 2009. p.1-24.

21. KESSLER, Gabriel. Sociología del delito amateur. Buenos Aires: Editorial Paidós, 2010.

22. KESSLER, Gabriel; DIMARCO, Sabina. Jóvenes, policía y estigmatización territorial en la periferia de Buenos Aires. Espacio abierto, v. 22, n. 2, p. 221-243, 2013.

23. MATZA, David; SYKES, Gresham. Juvenile delinquency and subterranean values. American Sociological Review, v. 26, n. 5, p.712-719, 1961.

24. MELO, Joice. Demografía de la juventud y derechos: los adolescentes en conflicto con la ley en el Brasil. Notas de Población, v. 37, n. 93, p. 7-36, 2011.

25. MERTON, Robert K. Estructura social y anomia. Revista Sociológica Americana, v. 3, n. 5, p. 672-682, 1938.

26. MISSE, Michel; MOTTA, Dilson. Crime: o social pela culatra. Rio de Janeiro: Achiamé, 1979.

27. RODRÍGUEZ, Ernesto. Juventud y violencia en América Latina: una prioridad para las políticas públicas y una oportunidad para la aplicación de enfoques integrados e integrales. Desacatos, n. 14, p. 36-59, 2004.

28. TENENBAUM, Gabriel. Vidas castigadas. Historias de adolescentes detectados por los sistemas de justicia de Montevideo y de Ciudad de México. Montevideo: Ediciones universitarias, Universidad de la República, 2020.

29. VEGA, Jesica. La ciudad y la violencia que experimentan los jóvenes. El caso de León, Guanajuato. In: ALVARADO, Arturo. Violencia juvenil y acceso a la justicia en América Latina. Tomo II. México: El Colegio de México, p. 113-164, 2014.

30. VERNAZZA, Lucía. Adolescentes y sistemas penales juvenil en América Latina: una reflexión a partir de la información cuantitativa disponible. Uruguay: Unicef, 2016.

31. WORLD PRISON BRIEF. An online database. PrisonStudies.org, 2021. Disponible en: https://www.prisonstudies.org

32. YLLESCAS, Jorge. Creer en la Santa Muerte durante el encierro. Ciudad de México: UNAM-Coordinación General de Estudios de Posgrado, 2019.

Recibido: 27 ago. 2021

Aprobado: 5 nov. 2021 
